\title{
Análisis de la relación entre los hábitos físico-saludables y la dieta con la obesidad en escolares de tercer ciclo de Primaria de la Provincia de Granada Analysis of the relationship between physical-healthy habits and diet with obesity in primary school students of the Province of Granada \\ José Iván Núñez-Quiroga, Félix Zurita-Ortega, Irwin Ramírez-Granizo, Antonio Miguel Lozano-Sánchez, Pilar Puertas-Molero, JoséLuis Ubago-Jiménez \\ Universidad de Granada (España)
}

Resumen. La obesidad y el sobrepeso se han convertido en uno de los trastornos más importantes de la actualidad. La aparición de este tipo de trastorno se debe a la influencia de factores como la dieta desequilibrada o la falta de actividad física, factores que provocan un aumento del IMC en escolares. Este estudio se realizó sobre 161 escolares de educación primaria, de edades comprendidas entre los 10 y 12 años y pertenecientes a la provincia de Granada. Se pretende analizar la relación existente entre los parámetros de la actividad física y la calidad de la dieta con los niveles de obesidad. Los resultados mostraron que la mayor parte de la población estudiada se encontraba dentro del normopeso y en una baja proporción los alumnos presentaban una dieta de baja calidad, sin haber diferencias significativas en cuanto al género. Por otra parte, el análisis de los datos mostró que el género masculino realiza mayor actividad física y por tanto presenta menos sedentarismo que el género femenino. Como conclusión, se demuestra la necesidad de motivar a la realización de deporte, sobre todo al género femenino, además de concienciar a los jóvenes de las consecuencias del sedentarismo y la obesidad.

Palabras clave. Obesidad, género, dieta, actividad física, educación primaria.

Abstract. Obesity and overweight have become one of the most important disorders nowadays. The onset of this type of disorder is due to the influence of factors such as unbalanced diet or lack of physical activity, factors that cause an increase in BMI in schoolchildren. This study was conducted on 161 schoolchildren of primary education, aged between 10 and 12 years old and belonging to the province of Granada. The aim is to analyze the relationship between physical activity parameters and diet quality with obesity levels. The results showed that participants were in a normal weight, whereas a low proportion of students presented a low-quality diet, with no significant differences by gender. On the other hand, the analysis of the data showed that males perform greater physical activity and therefore present less sedentary habits than females. In conclusion, the need to encourage students to carry out sports is demonstrated, especially in females. In addition, it is important to raise youth's awareness on health consequences of sedentary habits and obesity.

Keywords. Obesity, gender, diet, physical activity, primary education.

\section{Introducción}

La obesidad se ha convertido en una de las enfermedades más generalizadas en la sociedad del siglo XXI, con especial influencia en la población infantil (Chacón-Cuberos et al., 2017). De hecho, SaldivarCerón, Ramírez, Acevedo y Pérez-Rodríguez (2015) ya estimaron en su estudio que para finales de 2015 se darían más de 42 millones de casos de obesidad infantil en todo el mundo. Asimismo, y definiendo esta enfermedad, la Organización Mundial de la Salud(OMS) la concretó en 2012 como el exceso de grasa corporal que supone un riesgo para la salud.

Entre las principales causas que se asocian a esta epidemia, múltiples estudios hacen hincapié en el predominio de hábitos físico-saludables inadecuados, como pueden ser elevados niveles de sedentarismo o una dieta inadecuada (Sánchez-Cruz, Jiménez-Moleón, FernándezQuesada y Sánchez, 2013 o Martínez y Redecillas, 2011). En este sentido, resulta evidente la disminución de los niveles de práctica de actividad física en la población infantil, los cuales se ven acentuados, según Staiano y Calvert (2011), por una elevada prevalencia de ocio digital sedentario, por ello está ampliamente demostrada la importancia que tiene la práctica de actividad física en cualquier edad (Párraga, 2013) siendo beneficiosa a nivel fisiológico, psicológico y social (Sanz, 2017). Además, la actividad física en la sociedad actual es un tema elemental y prioritario, siendo un tema de especial relevancia en las políticas sociales y de salud (Zurita, Castro, Linares y Chacón, 2017).

En la actualidad, la sociedad se encuentra inmersa en la llamada «era digital» y autores como Trujillo, Muñoz y Villada (2013) muestran como los Exergames pueden hacer de la actividad física una práctica amena y gratificante con una gran jugabilidad, y todo ello aprovechando la motivación intrínseca que generan los videojuegos, mediante el empleo de plataformas como Wii de Nintendo, Kinect de Microsoft o Play

Fecha recepción: 02-11-17. Fecha de aceptación: 10-05-18 José Iván Núñez-Quiroga joseivan@correo.ugr.es
Station Move de Sony, adquiriendo la tecnología un peso importante dentro de la actividad física (Peng, Lin y Crouse, 2011 y Chacón et al., 2017) la cual serviría para combatir ese sedentarismo digital.

Además habría de tenerse en cuenta, un tercer factor fundamental a la hora de hablar de obesidad, como es el caso de la dieta. El modelo dietético predominante en nuestro país se desvincula cada vez más de la Dieta Mediterránea (DM), la cual es determinada por Alonso, Carranza, Rueda y Naranjo (2014) y Menéndez y Fernández (2015) como una de las dietas más saludables. En este sentido, el consumo de alimentos procesados con alto contenido en grasas y azucares refinados está ganando protagonismo en detrimento de aquellos que constituyen los pilares de una dieta saludable, como son la fruta, la verdura, las legumbres, los cereales o el pescado (Alonso et al., 2014). Dicha alimentación puede producir trastornos y enfermedades más allá del sobrepeso o la obesidad como son enfermedades de tipo cardiovascular, hipertensión, diabetes o problemas psicológicos (Estruch et al., 2013 o Esteves, Vieira, Bras, O’Hara y Pinheiro, 2017) entre otros.

El sedentarismo (de cualquier tipo) o inactividad física es uno de los principales orígenes del sobrepeso. En la actualidad, los videojuegos y otros dispositivos de pantalla se ha hecho un hueco importante en todos los hogares de nuestra sociedad, siendo su principal consumidor el sector infantil (Beltrán, Valencia y Molina, 2011). Este contexto, según Trujillo, Muñozy Villada (2013) y Espejo-Garcés et al., 2015, ha ocasionado que cada vez más se dedique menos tiempo a actividades lúdico-deportivas, disminuyendo considerablemente el gasto calórico diario; lo que unido a dietas de baja calidad se traduce en un aumento de los índices de sobrepeso.

Los elevados porcentajes de obesidad ligada a hábitos sedentarios en escolares y a la dieta de baja calidad basada, como se mencionó anteriormente, en alimentos procesados con alto contenido en grasas y azucares refinados, hace que este estudio persiga analizar las relaciones existentes entre estas variables. En esta línea, este trabajo persigue como principales objetivos:

Describir los niveles de obesidad, la dieta en el ámbito familiar y la práctica de actividad física en alumnos de tercer ciclo de primaria de un número determinado de centros públicos-concertados y urbanos de 
la provincia de Granada.

- Establecer la relación que existe entre los parámetros de actividad física y dieta con los niveles de obesidad en escolares de 10 a 12 años.

\section{Método}

\section{Diseño}

Se realizó un estudio no experimental, de tipo descriptivo y corte transversal. Asimismo, el diseño fue analítico y relacional, al concretar relaciones entre todas las variables.

El diseño fue mutltifactorial y multivariado, al concretar relaciones entre todas las variables.

\section{Participantes}

La muestra estuvo constituida por 161 estudiantes de Educación Primaria de la provincia de Granada, representados en un 60,9\% (N=98) por varones y en un 39,1\% ( $\mathrm{N}=63$ ) por mujeres, cuyas edades están comprendidas entre 10-12 años en el que el grueso de los participantes se encuentra entre los 10 (34,2\%) y los 11 años (48,4\%). La selección de los participantes se realizó a través de un muestreo aleatorio por conglomerados, considerando los escolares matriculados en tercer ciclo de Educación Primaria según centros los centros educativos que dieron la aceptación a formar parte del estudio, siendo estos públicos $(\mathrm{N}=3)$ y concertados $(\mathrm{N}=1)$.

\section{Instrumentosyvariables}

Se utilizó un cuestionario Ad Hoc, en el cual se incluyeron los aspectos sociodemográficos estudiados y aquellas variables relacionadas con la práctica de actividad física. Se consideraron las siguientes variables:

Práctica de actividad física fuera del ámbito escolar: Se considera el deporte que práctican los escolares, clasificados en 5 niveles de organización: $1=$ Deportes individuales sin contacto, $2=$ Deportes individuales con contacto, $3=$ Deportes colectivos sin contacto, $4=$ Deportes colectivos con contacto y $5=$ No practica deporte.

- Índice de Masa Corporal (IMC) categorizado, calculado mediante el método estandarizado propuesto por la OMS, el cual corresponde a la relación entre el peso expresado en kilos y el cuadrado de la altura, expresada en metros. Posteriormente, se realizó una codificación de los valores obtenidos en 1=Bajo peso, 2=Normopeso, 3=Sobrepeso y 4=Obesidad según el estudio realizado por Serrá-Majem et al. (2004).

- Adherencia a la Dieta Mediterránea: podremos analizar esta variable, en: 1= Dieta de baja calidad, 2= Dieta mejorable y 3= Dieta óptima.

Además se empleó:

Test KIDMED propuesto por Serra, Ribas, De la Cruz, Ortega, Pérez y Aranceta (2004), consiste en un cuestionario de 16 preguntas que deben responderse de manera afirmativa/negativa (sí/no). Las respuestas afirmativas en las preguntas que representan una connotación negativa en relación con la dieta mediterránea (son 4) valen -1 punto, y las respuestas afirmativas en las preguntas que representan un aspecto positivo en relación con la dieta mediterránea (son 12) valen +1 punto. Las respuestas negativas no puntúan. Por tanto, dicho índice puede oscilar entre-4 (mínima adherencia) y 12 (máxima adherencia). A la hora de representar que tipo de adherencia presenta cada individuo se realizará del siguiente modo: con valores comprendidos entre 8 y 12 (ambos inclusive) se entenderán como dieta optima, los valores situados entre 4 y 7 se entenderá como dieta mejorable y los valores comprendidos entre -4 y 3 se entenderán como dieta de baja calidad.

\section{Procedimiento}

En primer lugar se elaboró una carta informativa desde la Facultad de Ciencias de Educación de la Universidad de Granada con el fin de informar a los 7 centros educativos elegidos anteriormente de la naturaleza del estudio a realizar, así como solicitar la colaboración de los mismos. Posteriormente, los centros que aceptaron, en este caso 4 centros urbanos de los cuales 3 eran públicos y 1 concertado, participar contactaron con los tutores legales de los escolares, con el fin de obtener su consentimiento informado. Transcurrido este proceso, se procedió con la aplicación de los instrumentos. Esta etapa se realizó en horario lectivo, con la presencia de los investigadores -previamente instruidos en dos reuniones-, y del tutor de cada grupo, con el fin de asegurar la correcta aplicación de los instrumentos. Se han cumplimiento de las normas éticas de la Declaración de Helsinki de 1975 y se ha respetado el derecho de confidencialidad de los participantes. Asimismo, se ha informado a los centros educativos de los resultados obtenidos en la investigación.

\section{Análisis de los datos}

Para realizar el análisis de los datos se empleó el programa estadístico SPSS 22.0. Antes de comenzar a analizar las distintas variables fue necesario realizar el coeficiente alpha de Cronbach, siendo á=,704. Para el estudio de las variables se utilizaron descriptivos básicos; del mismo modo para la relación y comparación de los parámetros se emplearon tablas de contingencia, porcentajes y Chi-cuadrado de Pearson ( $p>$.05) para determinar la significatividad de las relaciones.

\section{Resultados}

Las variables analizadas fue el tipo de deporte que cada niño practicaba fuera de la escuela, como se puede observar, la mayor parte se encuentra dividida entre los niños practican algún deporte individual y sin contacto (31,7\%) y aquellos que practican deporte colectivo con contacto (38,5\%), aunque también hay que hacer mención a que el $20,5 \%$ no realiza ningún tipo de actividad física. Con respecto a la variable correspondiente a la obesidad, se ha de recalcar que el mayor porcentaje de la población (78,3\%) estaría en el rango del normopeso. Tal y como se puede apreciar a continuación, solamente el 9,3\%( $\mathrm{N}=15)$, de alumnos presentan una dieta de baja calidad. Por el contrario, el grueso de la población se encuentra repartido entre la dieta mejorable (42,2\%) y la dieta óptima (48,5\%), siendo esta última ligeramente superior (Tabla 1 ).

\begin{tabular}{|c|c|c|c|}
\hline \multicolumn{2}{|c|}{ Edad } & \multicolumn{2}{|c|}{ Sexo } \\
\hline 10 años & $34,2 \%(\mathrm{~N}=55)$ & Masculino & $60,9 \%(\mathrm{~N}=98)$ \\
\hline 11 años & $48,4 \%(\mathrm{~N}=78)$ & Femenino & $39,1 \%(\mathrm{~N}=63)$ \\
\hline 12años & $17,4 \%(\mathrm{~N}=28)$ & Total & $100,0 \%(\mathrm{~N}=161)$ \\
\hline \multicolumn{4}{|c|}{ Deporte } \\
\hline Indivi & Contacto & \multicolumn{2}{|c|}{$31,7 \%(\mathrm{~N}=51)$} \\
\hline Indivi & contacto & \multicolumn{2}{|c|}{$4,3 \%(\mathrm{~N}=7)$} \\
\hline Coles & contacto & \multicolumn{2}{|c|}{$5,0 \%(\mathrm{~N}=8)$} \\
\hline Colec & contacto & \multicolumn{2}{|c|}{$38,5 \%(\mathrm{~N}=62)$} \\
\hline & & \multicolumn{2}{|c|}{$20,5 \%(\mathrm{~N}=33)$} \\
\hline \multicolumn{4}{|c|}{ Obesidad } \\
\hline & & \multicolumn{2}{|c|}{$13,0 \%(\mathrm{~N}=21)$} \\
\hline & & \multicolumn{2}{|c|}{$78,3 \%(\mathrm{~N}=126)$} \\
\hline & & \multicolumn{2}{|c|}{$3,1 \%(\mathrm{~N}=5)$} \\
\hline & & \multicolumn{2}{|c|}{$5,6 \%(\mathrm{~N}=9)$} \\
\hline \multicolumn{4}{|c|}{ Dieta } \\
\hline Diet & calidad & \\
\hline \multicolumn{2}{|c|}{ Dieta mejorable } & \multirow{2}{*}{\multicolumn{2}{|c|}{$42,2 \%(\mathrm{~N}=68)$}} \\
\hline & & & \\
\hline
\end{tabular}

\section{Análisis relacional de la edad con el resto de las variables del estudio}

Como se puede apreciar a continuación (Tabla 2), tras el estudio relacional no se hallaron diferencias estadísticamente significativas entre la edad y el género ( $\mathrm{p}=, 436)$. Del mismo modo, tras analizar la relación entre edad y obesidad(Tabla 3), los resultados no mostraron diferencias estadísticamente significativas ( $\mathrm{p}=, 299$ ). En lo concerniente a la edad y la dieta (Tabla 4), tampoco se pudo establecer una asociación estadísticamente significativa ( $p=, 703)$. Respecto a la relación establecida entre la edad y el tipo de deporte o actividad física realizada, tal y como se puede apreciar (Tabla 5), no se observaron relaciones estadísticamente significativas ( $\mathrm{p}=, 748)$.

\section{Análisis relacional del género con el resto de las variables del estudio.}

Tal y como se expone a continuación (Tabla 6), no se apreciaron 
Tabla 2.

\begin{tabular}{|c|c|c|c|c|}
\hline \multirow{2}{*}{\multicolumn{2}{|c|}{ Edad }} & \multicolumn{2}{|c|}{ Sexo } & \multirow{2}{*}{ Total } \\
\hline & & Masculino & Femenino & \\
\hline \multirow{3}{*}{10 años } & Recuento & 33 & 22 & 55 \\
\hline & $\%$ Edad & $60,0 \%$ & $40,0 \%$ & $100,0 \%$ \\
\hline & $\%$ Sexo & $33,7 \%$ & $34,9 \%$ & $34,2 \%$ \\
\hline \multirow{3}{*}{11 años } & Recuento & 45 & 33 & 78 \\
\hline & \% Edad & $57,7 \%$ & $42,3 \%$ & $100,0 \%$ \\
\hline & \% Sexo & $45,9 \%$ & $52,4 \%$ & $48,4 \%$ \\
\hline \multirow{3}{*}{12 años } & Recuento & 20 & 8 & 28 \\
\hline & $\%$ Edad & $71,4 \%$ & $28,6 \%$ & $100,0 \%$ \\
\hline & \% Sexo & $20,4 \%$ & $12,7 \%$ & $17,4 \%$ \\
\hline \multirow{3}{*}{ Total } & Recuento & 98 & 63 & 161 \\
\hline & \% Edad & $60,9 \%$ & $39,1 \%$ & $100,0 \%$ \\
\hline & $\%$ Sexo & $100,0 \%$ & $100,0 \%$ & $100,0 \%$ \\
\hline
\end{tabular}

Tabla 3.

Distribución de la obesidad en función de la edad ( $\mathrm{p}=, 299)$.

\begin{tabular}{|c|c|c|c|c|c|c|}
\hline \multirow{2}{*}{\multicolumn{2}{|c|}{ Edad }} & \multicolumn{4}{|c|}{ Obesidad } & \multirow{3}{*}{$\begin{array}{c}\text { Total } \\
55\end{array}$} \\
\hline & & Bajo peso & Normopeso & Sobrepeso & Obeso & \\
\hline \multirow{3}{*}{10 años } & Recuento & 11 & 42 & 1 & & \\
\hline & $\%$ Edad & $20,0 \%$ & $76,4 \%$ & $1,8 \%$ & $1,8 \%$ & $100,0 \%$ \\
\hline & $\%$ Obesidad & $52,4 \%$ & $33,3 \%$ & $20,0 \%$ & $11,1 \%$ & $34,2 \%$ \\
\hline \multirow{3}{*}{11 años } & Recuento & 7 & 63 & $\frac{20,0}{2}$ & 6 & 78 \\
\hline & \% Edad & $9,0 \%$ & $80,8 \%$ & $2,6 \%$ & $7,7 \%$ & $100,0 \%$ \\
\hline & $\%$ Obesidad & $33,3 \%$ & $50,0 \%$ & $40,0 \%$ & $66,7 \%$ & $48,4 \%$ \\
\hline \multirow{3}{*}{12 años } & Recuento & 3 & 21 & 2 & 2 & 28 \\
\hline & $\%$ Edad & $10,7 \%$ & $75,0 \%$ & $7,1 \%$ & $7,1 \%$ & $100,0 \%$ \\
\hline & $\%$ Obesidad & $14,3 \%$ & $16,7 \%$ & $40,0 \%$ & $22,2 \%$ & $17,4 \%$ \\
\hline \multirow{3}{*}{ Total } & Recuento & 21 & 126 & 5 & 9 & 161 \\
\hline & $\%$ Edad & $13,0 \%$ & $78,3 \%$ & $3,1 \%$ & $5,6 \%$ & $100,0 \%$ \\
\hline & $\%$ Obesidad & $100,0 \%$ & $100,0 \%$ & $100,0 \%$ & $100,0 \%$ & $100,0 \%$ \\
\hline
\end{tabular}

Tabla 4.

Distribución de la edad en función a la adhesión a la dieta ( $\mathrm{p}=, 703)$.

\begin{tabular}{|c|c|c|c|c|c|}
\hline \multirow{2}{*}{\multicolumn{2}{|c|}{ Edad }} & \multicolumn{3}{|c|}{ Dieta } & \multirow{2}{*}{ Total } \\
\hline & & Dieta de baja calidad & Dieta mejorable & Dieta óptima & \\
\hline \multirow{3}{*}{10 años } & Recuento & 7 & 23 & 25 & 55 \\
\hline & $\%$ Edad & $12,7 \%$ & $41,8 \%$ & $45,5 \%$ & $100,0 \%$ \\
\hline & \% Dieta & $46,7 \%$ & $33,8 \%$ & $32,1 \%$ & $34,2 \%$ \\
\hline \multirow{3}{*}{11 años } & Recuento & 6 & 31 & 41 & 78 \\
\hline & $\%$ Edad & $7,7 \%$ & $39,7 \%$ & $52,6 \%$ & $100,0 \%$ \\
\hline & \% Dieta & $40,0 \%$ & $45,6 \%$ & $52,6 \%$ & $48,4 \%$ \\
\hline \multirow{3}{*}{12 años } & Recuento & 2 & 14 & 12 & 28 \\
\hline & \% Edad & $7,1 \%$ & $50,0 \%$ & $42,9 \%$ & $100,0 \%$ \\
\hline & $\%$ Die & $13,3 \%$ & $20,6 \%$ & $15,4 \%$ & $17,4 \%$ \\
\hline \multirow{3}{*}{ Total } & Recuento & 15 & 68 & 78 & 161 \\
\hline & $\%$ Edad & $9,3 \%$ & $42,2 \%$ & $48,4 \%$ & $100,0 \%$ \\
\hline & $\%$ Dieta & $100,0 \%$ & $100,0 \%$ & $100,0 \%$ & $100,0 \%$ \\
\hline
\end{tabular}

Tabla 5

Repartición de la edad en función del tipo de deporte practicado ( $\mathrm{p}=, 748)$.

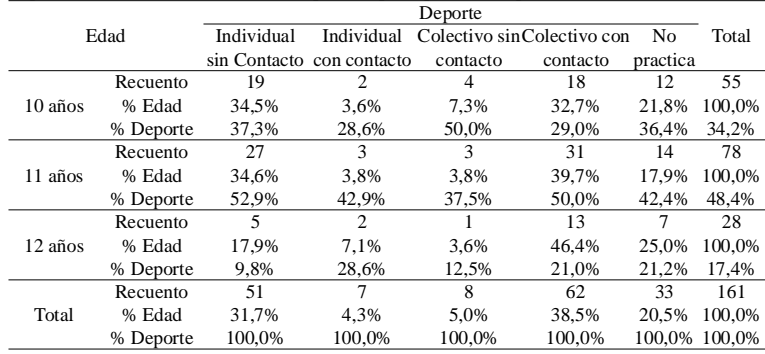

Tabla 6.

Relación entre el género y los tipos de obesidad ( $\mathrm{p}=, 075)$.

\begin{tabular}{ccccccc}
\hline \multirow{2}{*}{ Sexo } & \multicolumn{5}{c}{ Obesidad } & \multirow{2}{*}{ Total } \\
\cline { 3 - 6 } & & Bajo peso & Normopeso & Sobrepeso & Obeso & \\
\hline \multirow{3}{*}{ Masculino } & Recuento & 9 & 79 & 2 & 8 & 98 \\
& \% Sexo & $9,2 \%$ & $80,6 \%$ & $2,0 \%$ & $8,2 \%$ & $100,0 \%$ \\
& \% Obesidad & $42,9 \%$ & $62,7 \%$ & $40,0 \%$ & $88,9 \%$ & $60,9 \%$ \\
\hline \multirow{3}{*}{ Femenino } & Recuento & 12 & 47 & 3 & 1 & 63 \\
& \% Sexo & $19,0 \%$ & $74,6 \%$ & $4,8 \%$ & $1,6 \%$ & $100,0 \%$ \\
& \% Obesidad & $57,1 \%$ & $37,3 \%$ & $60,0 \%$ & $11,1 \%$ & $39,1 \%$ \\
\hline \multirow{3}{*}{ Total } & Recuento & 21 & 126 & 5 & 9 & 161 \\
& \% Sexo & $13,0 \%$ & $78,3 \%$ & $3,1 \%$ & $5,6 \%$ & $100,0 \%$ \\
& \% Obesidad & $100,0 \%$ & $100,0 \%$ & $100,0 \%$ & $100,0 \%$ & $100,0 \%$ \\
\hline
\end{tabular}

relaciones estadísticamente significativas $(\mathrm{p}=, 075)$ entre las variables de género y obesidad. Tampoco se encontraron diferencias estadísticamente significativas ( $\mathrm{p}=, 708)$, entre el género y adherencia a la dieta mediterránea (Tabla 7).

En contraposición y con lo que respecta a la relación estadística del género y el tipo de deporte practicado (Tabla 8), tal y como se puede observar a continuación, los resultados mostraron diferencias estadísticamente significativas $(p=, 000)$. Se pudo ver que el género femenino practica deportes del tipo individual sin contacto, en una proporción de dos niñas por cada niño, sin embargo, el género masculino práctica en mayor porcentaje $(55,1 \%)$ deportes de tipo colectivo con contacto. De forma general, el género masculino dedica un mayor tiempo a la práctica de actividad física, siendo el índice de participación
Tabla 7.

Asociación del género al tipo de adhesión a la dieta $(\mathrm{p}=708)$.

\begin{tabular}{|c|c|c|c|c|c|}
\hline \multirow{2}{*}{\multicolumn{2}{|c|}{ Sexo }} & \multicolumn{3}{|c|}{ Dieta } & \multirow{3}{*}{$\begin{array}{c}\text { Total } \\
98\end{array}$} \\
\hline & & Dieta de baja calidad & Dieta mejorable & Dieta óptima & \\
\hline \multirow{3}{*}{ Masculino } & Recuento & 10 & 39 & 49 & \\
\hline & \% Sexo & $10,2 \%$ & $39,8 \%$ & $50,0 \%$ & $100,0 \%$ \\
\hline & $\%$ Dieta & $66,7 \%$ & $57,4 \%$ & $62,8 \%$ & $60,9 \%$ \\
\hline \multirow{3}{*}{ Femenino } & Recuento & 5 & 29 & 29 & 63 \\
\hline & $\%$ Sexo & $7,9 \%$ & $46,0 \%$ & $46,0 \%$ & $100,0 \%$ \\
\hline & $\%$ Dieta & $33,3 \%$ & $42,6 \%$ & $37,2 \%$ & $39,1 \%$ \\
\hline \multirow{3}{*}{ Total } & Recuento & 15 & 68 & 78 & 161 \\
\hline & \% Sexo & $9,3 \%$ & $42,2 \%$ & $48,4 \%$ & $100,0 \%$ \\
\hline & \% Dieta & $100,0 \%$ & $100,0 \%$ & $100,0 \%$ & $100,0 \%$ \\
\hline
\end{tabular}

Tabla 8.

Relación entre el género y el tipo de deporte practicado $(p=, 000)$.

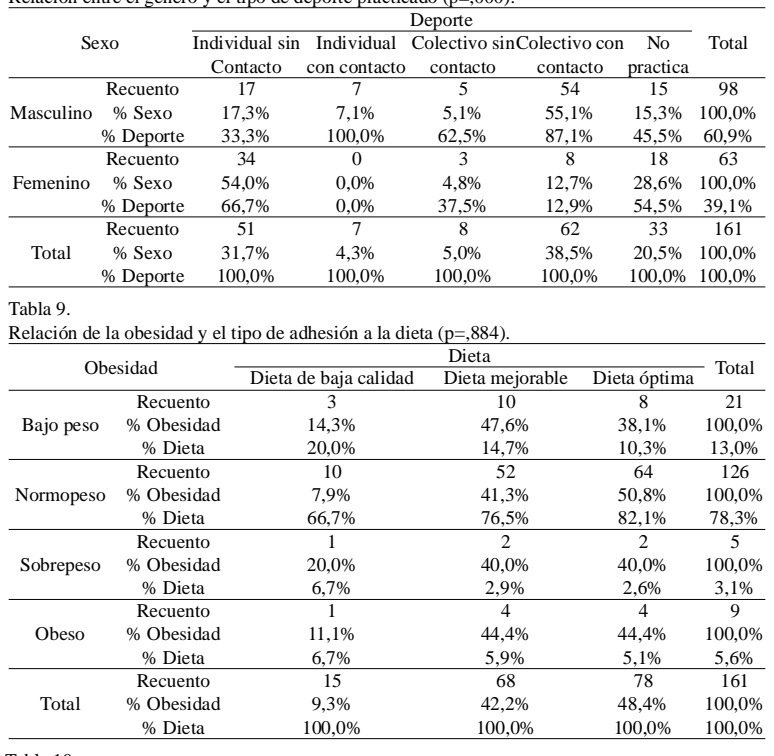

Tabla 10.

Correspondencia entre la obesidad y el tipo de deporte practicado $(\mathrm{p}=, 898)$.

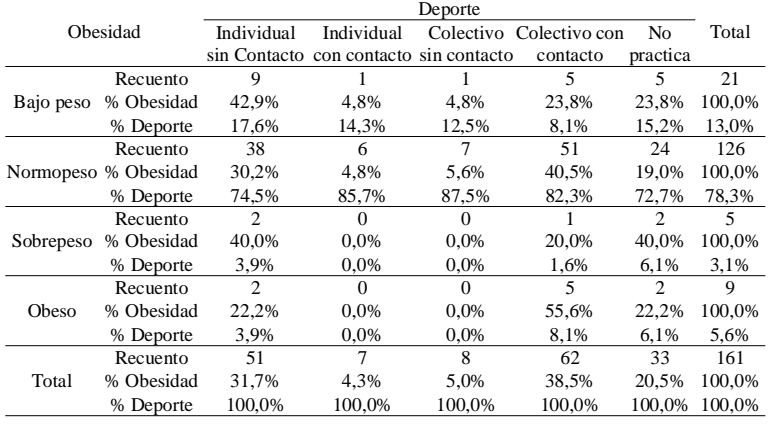

Tabla 11.

Distribución de la adhesión a la dieta en función del tipo de deporte practicado $(\mathrm{p}=, 334)$.

\begin{tabular}{|c|c|c|c|c|c|c|c|}
\hline \multirow{2}{*}{\multicolumn{2}{|c|}{ Dieta }} & & \multirow[b]{2}{*}{ Total } \\
\hline & & $\begin{array}{l}\text { Individual } \\
\text { sin Contacto }\end{array}$ & $\begin{array}{l}\text { Individual } \\
\text { con contacto }\end{array}$ & Colectivo sin & $\begin{array}{l}\text { Colectivo } \\
\text { con contact }\end{array}$ & $\begin{array}{c}\text { No } \\
\text { o practica }\end{array}$ & \\
\hline \multirow{3}{*}{$\begin{array}{l}\text { Dieta de baja } \\
\text { calidad }\end{array}$} & Recuento & 3 & 2 & 1 & 4 & 5 & 15 \\
\hline & \% Dieta & $20,0 \%$ & $13,3 \%$ & $6,7 \%$ & $26,7 \%$ & $33,3 \%$ & $100,0 \%$ \\
\hline & \% Deporte & $5,9 \%$ & $28,6 \%$ & $12,5 \%$ & $6,5 \%$ & $15,2 \%$ & $9,3 \%$ \\
\hline \multirow{3}{*}{$\begin{array}{l}\text { Dieta } \\
\text { mejorable }\end{array}$} & Recuento & 23 & 1 & 2 & 26 & 16 & 68 \\
\hline & \% Dieta & $33,8 \%$ & $1,5 \%$ & $2,9 \%$ & $38,2 \%$ & $23,5 \%$ & $100,0 \%$ \\
\hline & $\%$ Deporte & $45,1 \%$ & $14,3 \%$ & $25,0 \%$ & $41,9 \%$ & $48,5 \%$ & $42,2 \%$ \\
\hline \multirow{3}{*}{ Dieta óptima } & Recuento & 25 & 4 & 5 & 32 & 12 & 78 \\
\hline & \% Dieta & $32,1 \%$ & $5,1 \%$ & $6,4 \%$ & $41,0 \%$ & $15,4 \%$ & $100,0 \%$ \\
\hline & \% Deporte & $49,0 \%$ & $57,1 \%$ & $62,5 \%$ & $51,6 \%$ & $36,4 \%$ & $48,4 \%$ \\
\hline \multirow{3}{*}{ Total } & Recuento & 51 & 7 & 8 & 62 & 33 & 161 \\
\hline & $\%$ Dieta & $31,7 \%$ & $4,3 \%$ & $5,0 \%$ & $38,5 \%$ & $20,5 \%$ & $100,0 \%$ \\
\hline & \% Deporte & $100,0 \%$ & $100,0 \%$ & $100,0 \%$ & $100,0 \%$ & $100,0 \%$ & 6 100,0\% \\
\hline
\end{tabular}

del 60,9\% frente al 39,1\% del género femenino. Por último, resaltar el porcentaje de inactividad física también es ligeramente superior en el género femenino con un $28,6 \%$ frente al 15,3\% del género masculino.

\section{Análisis relacional de la obesidad con el resto de las variables del estudio}

En cuanto a la relación estadística establecida entre las siguientes dos variables de estudio, obesidad y adhesión a la dieta mediterránea (Tabla 9), no se apreció una relación estadísticamente significativa $(\mathrm{p}=, 884)$. En la siguiente tabla (Tabla 10) se muestran los valores medios obtenidos en función de la obesidad, y la relación que estos presentan frente al tipo de deporte practicado, tal y como se puede observar no se pudo obtener una relación estadística significativa ( $(p=, 898)$. 


\section{Análisis relacional entre la adhesión a la dietay el tipo de deporte practicado}

Por último se realizó un análisis relacional entre la adherencia a la dieta mediterránea con el tipo de deporte o actividad física practicada por parte de los participantes en el estudio (Tabla 11), de la cual no se pudo obtener un resultado significativo $(\mathrm{p}=, 334)$.

\section{Discusión}

El propósito de este estudio fue analizar las relaciones entre las habilidades físico-saludables y la dieta con los niveles de obesidad. Este estudio ha sido realizado sobre un total de 161 alumnos de 10 y 12 años. Las variables estudiadas, se asociaron a la calidad de vida que presentaba cada uno de los participantes, como es el caso de la dieta, la obesidad o la actividad física. De este modo, encontramos algunos estudios similares a este en el ámbito nacional e internacional (Sween, Wallington, Sheppard, Taylor, Llanos y Adams-Campbell, 2013; Martins, Honorio, Aldo, Costa, Batista y Cardoso, 2014 y Chacón, Zurita, Castro, Espejo, Martínez y Linares, 2016).

Tal y como se puede apreciar en los resultados, la primera relación establecida entre las variables de la edad y el género carecía de significatividad, esto es debido a que aunque la participación masculina sea ligeramente superior, no es suficiente para hablar de una relación, la cual sea relevante a la hora de realizar el estudio.

Posteriormente se estableció una relación estadística entre las variables de la edad y la obesidad, la cual siguiendo los parámetros establecidos por Serra, et al. (2003), siendo el porcentaje más alto el asociado al normopeso, lo cual nos indicó, que entre estas dos variables no se pudo establecer una relación directa, debido a la baja presencia, tanto del sobrepeso como de la obesidad, valores los cuales se encuentran por debajo de los obtenidos por otros autores como Meza, Sánchez, Bonilla y Jiménez (2016) o incluso los obtenidos por Serra et al. (2003) quienes revelan que en España el 13,9\% presenta problemas de obesidad, mientras que si se combina este problema con el de sobrepeso, asciende hasta el 26,3\% de la población estudiada.

Siguiendo con las distintas relaciones que se establecieron con la variable de la edad, se analizó dicha variable en función de la dieta. Tal y como se pudo apreciar en la tabla de resultados la mayor parte de la población estudiada se reparte casi de forma equitativa entre la una dieta mejorable y una óptima, resultados muy similares a los obtenidos por Velasco-Costa (2008) y Muntaner, Vidal, Cantallops, Borras y Palou (2017) en sus investigaciones y de igual modo careciendo de significatividad, lo que nos indica que la población escolar estudiada, presenta un patrón de adhesión a la dieta mediterránea aceptable casi en su totalidad pero no óptimo.

De manera consecutiva se estableció una relación estadística entre el género y la obesidad, como se ha podido observar anteriormente, tan solo 1 de cada 10 alumnos presentan obesidad coincidiendo con autores como Espejo-Garcés, Cabrera-Fernández, Castro-Sánchez, LópezFernández, Zurita-Ortega y Chacón-Cuberos (2015) o Pinel, Chacón, Castro, Espejo, Zurita y Pérez (2017). En contraposición a este hecho, algunos artículos fundamentan que más del $45 \%$ de los niños españoles padecen sobrepeso u obesidad (Morales-Falo, Sánchez-Moreno, Esteban, Alburquerque y Garaulet, 2013). Esto puede deberse a la zona geográfica y en el tiempo en el que ha sido tomada la muestra, además de tratarse de una etapa de mayor actividad física en los niños, realizada tanto dentro como fuera del centro escolar (Espejo-Garcés et al., 2015).

También podemos observar como la tasa de obesidad entre niños y niñas es bastante similar coincidiendo con autores como Zaleta (2013). En contraposición, autores como Kasier et al. (2014) alegan que los niños tienden a ser más obesos que las niñas, produciéndose así una nueva controversia.

Al observar la relación entre las variables del género y la adhesión a la dieta mediterránea, se pudo apreciar niveles muy similares entre niños y niñas, presentando 1 de cada 10 una dieta de baja calidad. Dichos datos presentan similitudes con los obtenidos en otros estudias realiza- dos como es el caso de Pinel et al. (2017), esto puede deberse a la zona geográfica donde se estudió la muestra, cuya proximidad al mediterráneo favorece la adhesión a este tipo de dieta.

Por otra parte y siguiendo con los datos obtenidos, 5 de cada 10 niñas practican deporte de carácter individual y sin contacto, por el contrario la realización de actividad física por parte de los niños se centra en el deporte colectivo con contacto, el cual practican 6 de cada 10 niños, esto puede deberse, como ya se ha comentado anteriormente, a una etapa de mayor actividad física en los escolares. Por otra parte, la práctica de estos tipos de deporte tanto de niños como de niñas puede deberse a la tendencia social, en la cual predominan en el género masculino deportes como el futbol o la gimnasia rítmica en el género femenino. Del mismo modo y en consonancia con otros estudios realizados por autores como Mauro-Martín et al. (2014), quienes afirman en sus investigaciones que el género masculino es más propenso a la realización de actividad física que el femenino, esto se ve confirmado con los datos obtenidos del estudio realizado, en el que el género femenino presenta un nivel de inactividad física o sedentarismo de 3 de cada 10 , siendo ligeramente superior al masculino.

Por último, al observar las relaciones establecidas entre la obesidad y la dieta y la obesidad y la actividad física, se puede entender que no se encontraran diferencias significativas, pues como se muestra anteriormente en las correspondientes tablas, los niveles más bajos se detectaron el alumnos con obesidad y con una dieta de mala calidad encontrándose en una proporción de 1 de cada 10 . Del mismo modo se observóla relación entre alumnos con normopeso y que realizan algún tipo de práctica deportiva siendo 8 de cada 10 , o por el contrario la presencia de sobrepeso u obesidad relacionada con el sedentarismo encontrándose ambas en una proporción de 1 de cada 10. Al hilo de autores como Dattilo, Birch, Krebs, Lake, Traveras y Saavedra (2012).

\section{Conclusiones}

En relación a lo expuesto, este estudio alcanza como principales conclusiones:

Los estudiantes de Granada, cuya edad está comprendida entre 10 y 12 años, presentan en una proporción de 8 de cada 10 un peso normal. Por otra parte, debemos tener en cuenta la dieta, resaltando la dieta óptima, la cual se encuentra en una proporción de 5 de cada 10, esto nos indica que actualmente la población infantil (10 a 12 años) realiza una alimentación saludable basada en la dieta mediterránea.

También cabe resaltar la participación o realización de los alumnos en algún tipo de actividad física, la práctica de deporte colectivo con contacto predomina ligeramente sobre la práctica de deporte individual sin contacto. Además, como dato significativo, pocos alumnos presentaron inactividad física.

Al establecer una relación estadística entre la obesidad, la dieta y la actividad física y teniendo en cuenta el género de los participantes, se pudo observar que ambos géneros presentarían igualdad estadística en dieta óptima (5 de cada 10) y un IMC de peso normal, siendo el masculino (8 de cada 10) ligeramente superior al femenino (7 de cada 10), pero en lo que ha realización de actividad física se refiere de manera general, el sexo masculino presenta una mayor disposición a la realización de la misma (9de cada 10). De una manera más específica se puede observar que el género femenino tiene predilección por deportes individuales sin contacto, mientras que el masculino prefiere los deportes colectivos con contacto.

\section{Perspectivas futuras y limitaciones}

Las limitaciones que se encontraron a la hora de realizar este estudio fueron principalmente, la falta de estudios realizados sobre este tema en niños de 10 a 12 años, lo que supone una falta de información a la hora de contrastar los datos obtenidos con los de otros autores, debido a que la mayoría de trabajos están centrados en la población adolescente o adulta. Sin embargo, haber centrado el estudio en edades más tempra- 
nas, nos puede dar una idea más aproximada a la realidad en cuanto al estilo de vida que llevan los alumnos de educación primaria, esto puede considerarse uno de los aspectos más importantes de la investigación, puesto que sirve para mostrar ciertas diferencias o aspectos relevantes, que muchas veces pasan por alto. Por otra parte, al pertenecer los individuos a educación primaria, se encontraron ciertas dificultades en la realización del test, debido a ciertas indecisiones en la selección de la respuesta. Aun así, los instrumentos empleados se encontraban adaptados a esta población para poder obtener unos datos más fiables de los que se obtendrían utilizando pruebas modificadas para adultos.

Para finalizar, como se ha podido comprobar, el género masculino tiene una mayor predisposición a llevar unos hábitos de vida saludables, esto nos indica que se debe fomentar y motivar al género femenino a participar y realizar más actividad física de cualquier tipo. Por otra parte, y siendo uno de los temas sociales más importantes, sería necesario llevar a cabo una serie de intervenciones para concienciar a los niños de las consecuencias que tiene llevar una vida sedentaria y poco saludable en términos de alimentación.

\section{Referencias}

Alonso, F.J., Carranza, M.D., Rueda, J.D. y Naranjo, J. (2014). Composición corporal en escolares de primaria y su relación con el hábito nutricional y la práctica reglada de actividad deportiva. Revista Andaluza de Medicina del Deporte, 7(4), 137-142.

Beltrán, V. J., Valencia, A. y Molina, J.P. (2011). Los videojuegos activos y la salud de los jóvenes: revisión de la investigación. Revista Internacional de Medicina y Ciencias de la Actividad Física y Deporte, 10(41), 203-219.

Chacón, R., Arufe, V., Espejo, T., Chacón, J., Zurita, F. y Castro, D. (2017) Práctica físico-deportiva, actividades de ocio y conceoción sobre la Educación Física en escolares de ACoruña. Revista Retos: Nuevas tendencias en Educación Física, Deporte y Recreación, 32, 163-166

Chacón, R., Zurita, F., Castro, M., Espejo, T., Martínez-Martínez, A. y Linares M. (2016). Estudio sobre la aplicabilidad de exergames para la mejora de los índices de obesidad y la imagen corporal en escolares. Revista Iberoamericana de Psicología del Ejercicio y el Deporte, 11(1), 97-105.

Dattilo, A.M., Birch, L., Krebs, N.F., Lake, A., Traveras, E.M. y Saavedra, J.M. (2012). Need for early interventions in the prevention of pediatric overweight: a review and upcoming directions. Journal of obesity, 22, 1-18.

Espejo-Garcés, T., Cabrera-Fernández,A., Castro-Sánchez, M., López-Fernández, J.F., Zurita-Ortega, F. y Chacón-Cuberos, R. (2015). Modificaciones de la obesidad a través de la implementación de herramientas físico-posturales en escolares. Revista Retos: Nuevas tendencias en Educación Física, Deportey Recreación, 28, 78-83.

Espejo-Garcés, T., Cabrera-Fernández, Á., Castro-Sánchez,M.,López-Fernández, J. F., Zurita-Ortega, F., y Chacón-Cuberos, R. (2015). Modificaciones de la obesidad a través de la implementación de herramientas físico-posturales en escolares. Retos. Nuevas Tendencias en Educación Física, Deporte y Recreación, 28, 78-83.

Esteves, D., Vieira, S., Brás, R., O’Hara, K., \& Pinheiro, P. (2017). Nível de atividade física e hábitos de vida saudável de universitários portugueses. Revista Iberoamericana de Psicología del Ejercicio yel Deporte, 12(2), 261-270.

Esteves, D., Vieira, S., Brás, R., O’Hara, K., \& Pinheiro, P. (2017). Nível de atividade física ehábitos de vida saudável de universitários portugueses. Revista Iberoamericana de Psicología del Ejercicio yel Deporte, 12(2), 261-270.

Estruch, R., Ros, E., Salas-Salvadó. J., Covas, M. I., Corella, D., Arós, F., ... y Lamuela-Raventos, R. M. (2013). Primary prevention of cardiovasculardisease with a Mediterranean diet. New England Journal of Medicine, 368(14), 127990. doi:10.1056/NEJMoal200303.

González, J. y Portolés, A. (2016). Recomendaciones de actividad física y su relación con el rendimiento académico en adolescentes de la Región de Murcia. Retos: Nuevas Tendencias EnEducación Física, Deporte Y Recreación, 29, 100-104.

Kaiser, L.L.,Aguilera, A.L., Horowitz, M., Lamp, C., Johns, M., Gomez-Camacho, R., Ontai, L. y De la Torre, A. (2014). Correlates of food patterns in Young Latino children at high risk of obesity. Public Health Nutrition, 18(16), 30423050.

Martínez-López, E.J. y Redecillas-Peiró, M.T. (2011). Prevalencia de sobrepeso y obesidad en escolares de la provincia de Jaén. Revista Internacional de Medicina y Ciencias de la Actividad Física y el Deporte, 11(43), 472-490.

Martínez-López, E.J. y Redecillas-Peiró, M.T. (2011). Prevalencia de sobrepeso y obesidad en escolares dela provincia de Jaén. Revista Internacional de Medicina y Ciencias de la Actividad Física y el Deporte, 11(43), 472-490.

Martins, J., Honório, S., Costa, A., Batista, M. y Cardoso, J. (2014). Longitudinal study of physical fitness levels, BMI and Childhood obesity in school context. Journal of Human Sport and Exercise, 9(2), 645-655.

Mauro-Martín, I., Garicano-Villa, E., González-Fernández, M., Villacorta-Pérez,P, Megias-Gamarra,A., Miralles-Rivera, B., Figueroa-Borque, M.,Andrés-Sánchez, N., Bonilla-Navarro, M.A., Arranz-Poza, P., Bernal-Maurandi, M.D., RuizLeón, A.M., Moraleda-Ponzol, E. y de la Calle-dela Rosa, L. (2014). Hábitos alimentarios y psicológicos en personas que realizan ejercicio físico. Nutrición Hospitalaria, 30(6), 1324-1332.

Menéndez-Santurio, J. I., \& Fernández-Río, J. (2015). Hábitos de alimentación y actividad física de Menores Extranjeros No acompañados (MENAs): un estudio decaso. Retos. Nuevas tendencias en Educación Física, Deportey Recreación, 28, 33-39.

Meza, R., Sánchez, F., Bonilla, R y Jiménez, C., (2016). Prevalencia del sobrepeso y obesidad en niños deEscuelas de Santo Domingo de Heredia, Costa Rica. Rev Hisp Cienc Salud. 2(1), 12-20.

Morales Falo, E.M.,Sánchez Moreno, C., Esteban, A.,Alburquerque, J.J. y Garaulet, M. (2013).Calidad de la dieta «antes y durante» un tratamiento de pérdida de peso basado en dieta mediterránea, terapia conductual y educación nutricional. Nutrición Hospitalaria, 28(4), 980-987.

Muntaner-Mas, A., Vidal-Conti, J., Cantallops, J. Borras, P. y Palou, P. (2017) Obesity and physical activity patterns among Balearic Islands children and adolescents: a cross-sectional study. Journal of Human Sport and Exercise, 12(2),333-348.

Organización Mundial de la Salud (Mayo de 2012). Obesidad y sobrepeso. Nota descriptiva $\mathrm{N}^{\circ}$ 311. Recuperado el día 10 de julio del 2015, desde: http:// www.who.int/mediacentre/factsheets/s311/es/

Párraga, J. A. (2013). Importancia de la práctica de actividad física. EmásF, Revista Digital de Educación Física. 20, 4-7.

Peng, W., Lin, J. y Crouse, J. (2011). Is Playing Exergames Really Exercising? A Meta-Analysis of Energy ExpenditureinActive Video Games. Cyberpsychology, Behavior, and Social Networking, 14(11), 681-688.

Pinel, C., Chacón, R., Castro, M., Espejo, T., Zurita, F. y Cortés, A. (2017) Diferencias de género en relación con el Índice de Masa Corporal, calidadde la dieta y actividades sedentarias en niños de 10 a 12 años. Retos, 31, 176-180.

Saldivar-Cerón, H.I., Ramírez, A.G.,Acevedo, M.A. y Pérez-Rodríguez, P. (2015). Obesidad infantil: factor de riesgo para desarrollar pieplano. Boletín Médico del Hospital Infantil de México, 72(1), 55-60.

Sánchez-Cruz, J.J., Jiménez-Moleón, J.J., Fernández-Quesada, F.y Sánchez, M.J. (2013). Prevalencia de obesidad infantil y juvenil en España en 2012. Revista española de cardiología, 66(5), 371-376.

Sanz, D. (2017). Niveles de actividad física moderada-vigorosa de adolescentes del municipio de Soria. Sportis Sci J, 3(1), 100-122.

Serra, Ll., Ribas, L., Aranceta, J., Pérez, C., Saavedra, P. y Peña, L. (2003). Obesidad infantil y juvenil en España. Resultados del Estudio enKid (19982000). Medicina Clinica (Barc), 121(19), 725-732.

Serra, Ll., Ribas, L., de la Cruz, J., Ortega R., Pérez, C. y Aranceta, J.(2004) Alimentación, jóvenes y dietamediterránea en España. Desarrollo del KIDMED, índice de calidad de la dieta mediterránea en la infancia y la adolescencia. In: Serra Majem L, Aranceta Bartrina J, editores. Alimentación infantil y juvenil. Masson; 2004(reimpresión). p. 51-59.

Serrá-Majem, L., Ribas, L., Ngo, J., Ortega, R.M., García, A., Pérez-Rodrigo, C., et al. (2004). Food, youth and the Mediterranean diet in Spain. Development of KIDMED, Mediterranean diet quality index in children and adolescents. Public Health Nutrition, 7(7), 931-935.

Staiano, A.E. y Calvert, S. L. (2011). Exergames for physical education courses: Physical, social, and cognitive benefits. Child development perspectives, 5(2), 93-98.

Sween J., Wallington, S., Sheppard, V., Taylor, T., Llanos, A. y Adams-Campbell, L. (2013). The role of exergaming in improving physical activity. Journal of Physical Activity and Health, 11(4), 864-870.

Trujillo, J. Muñoz, J. y Villada, J. (2013). Exergames: una herramienta tecnológica para la actividad física. Revista Médica de Risaralda, 19(2), 126-130.

Velasco-Costa, J. (2008). Evaluación de la dieta en escolares de Granada. Granada: Tesis Doctoral.

Zaleta, L. (2013). Aplicación de un programa de ejercicio físico para mejorar la postura corporal en escolares de 9 a 12 años de Ciudad del Carmen (México). Granada:Tesis Doctoral.

Zurita, F., Castro, M., Linares, M., Chacón, R. (2017). Resiliencia, un elemento de prevención en actividad física. Sportis, 3(1), 50-62. 